\author{
Maj VIVEK SRIVASTAVA", Lt Col RAJAT KUMAR ${ }^{+}$
}

MJAFI 1997; $53:$ 235-236

G rowth factors are cytokines that regulate growth and differentiation of blood cells. Many of these factors are now available in large quantities for clinical trials and therapy.

\section{Erythropoietin (Epo)}

Epo stimulates the erythroid colony forming unit (CFU-E) and the burst forming unit erythroid (BFU-E), resulting in increased formation of heme in nucleated erythroid cells, and early release of reticulocytes from marrow. Epo has been used to treat anaemia in chronic renal failure [1]. Recently it has also been approved for zidovudine (AZT) induced anaemia [2] and with cancer chemotherapy [3]. It is being tried in several other diseases [4]. Hypertension can occur if haematocrit rises too rapidly in dialysis patients. Iron deficiency anaemia may be precipitated and can be prevented by iron supplements.

\section{Granulocyte/Macrophage Colony Stimulating Factor (GM-CSF)}

GM-CSF acts synergistically with Interleukin-3 on several cell line precursors. It increases the cytotoxicity and phagocytosis of mature granulocytes and also reduces the motility and clearance of granulocytes from the peripheral circulation. It has been used in patients with haematological malignancies who are undergoing chemotherapy or bone marrow transplantation [5].

It reduces the incidence of infection in neutropenia due to myelotoxic chemotherapy. A dose of $3-10 \mu \mathrm{g} / \mathrm{kg} /$ day is given for $7-10$ days. GM-CSF has also being tried in patients with aplastic anaemia, and myelodysplastic syndromes, and congenital cyclic neutropenia [6].
Important side effects include fever, myalgia, skin rash, pericarditis, pleural effusions, and worsening of prior liver or kidney disease.

\section{Granulocyte Colony Stimulating Factor (G-CSF)}

G-CSF stimulates precursor cells and shortens the time required for maturation and release into the circulation. It decreases the incidence of infection in patients who receive myelotoxic chemotherapy. G-CSF therapy has been shown to reduce the number of neutropenic days in patients undergoing chemotherapy [7] and in patients with bone marrow transplantation [8]. It is given in a dose of $5 \mu \mathrm{g} / \mathrm{kg} /$ day for $7-10$ days. Bone pain is the commonest adverse effect and responds to simple analgesics.

\section{Multi-CSF/Interleukin-3 (IL-3)}

Multi-CSF stimulates colony formation in most of the haematopoietic cell lines. Besides its synergistic action with GM-CSF also expands the BFUE compartment in association with Epo. MultiCSF significantly shortens post-chemotherapy neutropenia. Toxic reactions include fever, flushing, headache, and local erythema.

PIXY-321 is a genetically engineered protein combining IL-3 and GM-CSF. Several studies have demonstrated greater improvement after use of this cytokine with high dose chemotherapy [9].

\section{Thrombopoietin (TSF)}

Thrombopoiesis stimulating factor (TSF) or thrombopoietin is a factor responsible for growth and maturation of platelets. A recent study showed that a truncated version of TSF lessened the severity and duration of platelet nadir after 
chemotherapy [10]

\section{Colony Stimulating Factor-1 (CSF-1 or M-CSF)}

CSF-1 was one of the first colony stimulating factors to be discovered that predominantly affected the myeloid lineage. It stimulates monocytes/macrophage colony alone and acts synergistically with GM-CSF and IL-3. Unfortunately, thrombocytopenia is a common side-effect of CSF-1 infusion despite the increase of megakaryocytes in bone marrow. The enhancement of macrophage function may prove useful in enhancing the immune system.

It can therefore be concluded that haematopoietic growth factors are useful in many fields of clinical medicine. However, caution is recommended in view of reports of accelerated transformation of myelodysplastic syndrome to acute leukaemia [11].

\section{REFERENCES}

1. Eschbach JVV, Kelly MR, Haley NR, et al. Treatment of anemia of progressive renal failure with recombinant huInan erythropoietin. N Engl J Med 1989; 321 : 158-63.

2. Goodnough LT, Anderson KC, Kurtz $S$, et al. Indications and guidelines for the use hematopoietic growth factors. Transfusion. 1993; $33: 944-59$.

3. Spivak JL. Recombinant human erythropoietin and the anemia of cancer. Blood 1994; 84 : 997-1004.

\section{Drug Test Report}

Two out of 11 ampicillin brands topped the overall ratings based on the comparative testing, ranking and evaluation conducted in our laboratory. They also gave the most value for money. Ampilin from Lyka came out with the highest score of 64.07 per cent. It was also the least expensive at Rs 2.40 per capsule. The next in line, Broadicilin from Alkem had a score of 62.1 per cent, and cost Rs 2.79 per capsule. The lowest position went to Silver's Ampicillin capsules (33.48\%), which was also the most expensive at Rs 4.00 per capsule.

For a drug considered to be safe even for children, it was shocking to find two failed brands in the market. Ampicillin from Shifa Pharmaceuticals failed in the assay (the amount of active ingredient in the drug) test. If a capsule/tablet does not contain the required amount of antibiotic, it would lead to ineffective therapy, and may result in the development of a resistant strain. And Depilin from Dey's Medical Stores did not meet the requirements for uniformity of weight. This indicates that Good Manufacturing Practices (GMP) are not followed by the manu-
4. Means RT Jr, Krantz SB. Progress in the understanding of the pathogenesis of anemia of chronic disease. Blood $1992 ; 80: 1639-47$.

5. Parikh PM, Kulkarni S, Kapoor G, et al. Role of growth factors in hastening hematopoietic recovery following HLA matched sibling allogenic bone marrow transplantation. J Assoc Physicians India 1995; 404-5

6. Antin JH, Smith BR, Holnes W, et al. Phase I/II study of recombinant human GM-CSF in aplastic anemia and myelodysplastic syndrome. Blood 1988; 72 : 705-13.

7. Crawford J, Ozer H, Stoller R, et al. Reduction by G-CSF of fever and neutropenia by chemotherapy in patients with small cell lung cancer. N Engl J Med 1991; 325 : 164-70.

8. Weisdorf DJ, Verfaillie CM, Davies $S M$, et al. Hematopoietic growth factors for graft failure after bone marrow transplantation : a randomized trial of GM-CSF versus sequential GM-CSF plus G-CSF. Blood 1995; 86 : 3452-6.

9. O'Shaughnessy JA, Venzon DJ, Gossard M, et al. A phase 1 study of sequential versus concurtent interleukin-3 and CM-CSF factor in advanced breast cancer patients treated with FLAC (5-fluorouscil, leucovorin, doxorubicin, cyclophosphamide) chemotherapy. Blood 1995; 86 : 2913-9.

10. Fanucchi M, Glaspy J, Crawfood J, et al. Effects of polyethylene-glycol conjugated recombinant human megakaryocyte growth and development factor on platelet counts after chemotherapy for lung cancer. N Fngl J Med 1997; 336 : 404-9.

11. Rajat Kumar, Ranga Rao R, Rajan S. Hematopoietic growth factors in hematological malignancies. J Assoc Physicians India 1995: Suppi 3 : 5-10.

facturer, as the brand does not adhere to the specific standards set down by I.P.

All the brands were well within the limits for both dissolution and disintegration.

There were quite a few borderline results in uniformity of weight and assay, which indicate the manufacturers' tendency to somehow comply with the statutory provisions, however marginally. The brands which showed such results were : Biocilin of Biochem Pharma and Ampicillin of Aesculapius Remedies in Uniformity of Weight; Roscillin of Ranbaxy, Bacipin of Alembic and Ampicillin of Silver Pharma in Assay. These brands may have less effect in a patient than the other passing brands which follow good manufacturing practices.

(The above Key Findings are from a Test Report on Ampicillin published by the Consumer Education and Research Socieny. Ahmedabad in their journal Consumer Confrontation 1997; 17: 2l-5. MAFI is grateful for permission to use the report.) 